Article

\title{
Coincidence Points of a Sequence of Multivalued Mappings in Metric Space with a Graph
}

\author{
Muhammad Nouman Aslam Khan ${ }^{1,2,+}$, Akbar Azam ${ }^{2, \dagger}$ and Nayyar Mehmood ${ }^{3, *,+}$ \\ 1 School of Chemical and Materials Engineering, National University of Sciences and Technology, H-12, \\ Islamabad 44000, Pakistan; mnouman@scme.nust.edu.pk \\ 2 Department of Mathematics, COMSATS Institute of Information Technology, Chak Shahzad, \\ Islamabad 44000, Pakistan; akbarazam@yahoo.com \\ 3 Department of Mathematics and Statistics, International Islamic University, H-10, Islamabad 44000, Pakistan \\ * Correspondence: nayyar.mehmood@iiu.edu.pk; Tel.: +92-51-9019-948 \\ + These authors contributed equally to this work.
}

Academic Editor: Lokenath Debnath

Received: 24 March 2017; Accepted: 22 May 2017; Published: 26 May 2017

\begin{abstract}
In this article the coincidence points of a self map and a sequence of multivalued maps are found in the settings of complete metric space endowed with a graph. A novel result of Asrifa and Vetrivel is generalized and as an application we obtain an existence theorem for a special type of fractional integral equation. Moreover, we establish a result on the convergence of successive approximation of a system of Bernstein operators on a Banach space.
\end{abstract}

Keywords: graphic contraction; coincidence points; sequence of multivalued maps; Bernstein operators

\section{Introduction and Preliminaries}

For the metric space $(X, d)$, using the notions of Nadler [1] and Hu [2], denote $C B(X), C(X)$ and $2^{X}$ by the collection of nonempty closed and bounded, compact and all nonempty subsets of $X$ respectively. Consider $A, B \in C B(X)$ the distance between sets $A$ and $B$ is defined by $d(A, B)=\inf _{x \in A, y \in B} d(x, y)$, which does not allow to enjoy the properties of metric on $C B(X)$ therefore a well known idea of Hausdorff-Pompeiu distance $H$ on $C B(X)$ induced by $d$ is used to define a metric on $C B(X)$ as follows:

$$
H(A, B)=\inf \{\epsilon>0: A \subseteq N(\epsilon, B), B \subseteq N(\epsilon, A)\},
$$

where:

$$
N(\epsilon, A)=\{x \in X: d(x, a)<\epsilon, \text { for some } a \in A\} .
$$

In 1969, Nadler [1] proved fixed point results for multivalued mappings in complete metric spaces, using the Hausdorff distance $H$, which was the generalization of Banach contraction principle in the settings of set-valued mappings. Covitz and Nadler [3] extended the idea of multivalued mappings in the generalized metric spaces. Reich [4] in 1972 published a fixed point result for the multivalued maps on the compact subsets of a complete metric space and posed the question, "can $C(X)$ be replaced by $C B(X)$ ?". In 1989, Mizoguchi and Takahashi answered this question in Theorem 5 of [5] and they also provide some Caristi type theorems for multivalued operators. Whereas $\mathrm{Hu}$ [2] in 1980 extended the multivalued fixed point results from complete metric space to complete $\varepsilon$-chainable metric space. Azam and Arshad [6] have extended the Theorem 6 of [1] by finding the fixed points of a sequence of locally contractive multivalued maps in $\varepsilon$-chainable metric space. Further Feng and Liu [7] used 
the concept of lower semi-continuity and a generalized contractive condition to extend the result of Nadler [1] and Caristi type theorems as defined in [5]. For more references the readers are referred to the work of Ciric [8], Klim and Wardowski [9,10] , Nicolae [11].

Jachymski [12] in 2007 unified and extended the work of Nieto [13] and Ran and Reuring [14] by defining a new class of contractions (G-contraction ) on metric space $(X, d)$ endowed with a graph. The connectivity of the graph brings more attractions regarding a necessary and sufficient condition for any G-contractive operator to be a Picard operator.

In the present article, fascinated by [6] the existence of coincidence points of a sequence of multivalued maps with a self map are taken into account with a generalized form of G-contraction. This provides a new way to generalize many existing results in the literature (see $[1,6]$ and the references therein).

Let us recall some definitions from graph theory with the perspective of using them in our ideas and results. For a metric space $(X, d)$ let $\triangle$ be the diagonal of the Cartesian product $X \times X$. Consider a directed graph $G$ such that $X=V(G)$, where $V(G)$ is the set of vertices of $G$. The set $E(G)$ of edges of $G$ contains all the loops. If $G$ has no parallel edge then we can identify $G$ with the pair $(V(G), E(G))$. Further, the graph $G$ can be dealt with as a weighted graph if each edge is assigned by the distance between its edges.

Consider a directed graph $G$, then $G^{-1}$ denote the graph obtained from $G$ by reversing the direction of edges and if we ignore the direction of edges in graph $G$ we get an undirected graph $\tilde{G}$. The pair $\left(V^{\prime}, E^{\prime}\right)$ is said to be a subgraph of $G$ if $V^{\prime} \subseteq V(G)$ and $E^{\prime} \subseteq E(G)$ and for any edge $(a, b) \in E^{\prime}$ for all $a, b \in V^{\prime}$.

Recall some fundamental definitions regarding the connectivity of graphs, which can be found in [15].

Definition 1. A path in $G$ from the vertex $p$ to $q$ of length $K$, is a sequence $\left\{p_{i}\right\}$ of $K+1$ vertices such that $p_{0}=p_{1}, \ldots, p_{K}=q$ and $\left(p_{j-1}, p_{j}\right) \in E(G)$ for $j=1,2, \ldots, K$.

Definition 2. A graph $G$ is called connected if there is a path between any two vertices. Graph $G$ is weakly connected if $\tilde{G}$ is connected.

Definition 3. For $a, b$ and $c$ in $V(G),[a]_{G}$ denote the equivalence class of the relation $\sim$ defined on $V(G)$ by the rule:

$$
b \sim c \text { if there is a path in } G \text { from } b \text { to } c .
$$

For $v \in V(G)$ and $K \in \mathbb{N} \cup\{0\}$ by $[v]_{G}^{K}$ we denote the set

$$
[v]_{G}^{K}:=\{u \in V(G) \text { : there is a path of length } K \text { from } v \text { to } u\} .
$$

Following is the definition of G-contraction by Jachymski [12].

Definition 4. [12] Let $(X, d)$ be a metric space endowed with a graph $G$. We say that a mapping $T: X \rightarrow X$ is a $G$-contraction if $T$ preserves edges of $G$ i.e.,

$$
\underset{x, y \in X}{\forall}(x, y) \in E(G) \Rightarrow(T x, T y) \in E(G),
$$

and there exists some $\alpha \in[0,1)$ such that:

$$
\underset{x, y \in X}{\forall}(x, y) \in E(G) \Rightarrow d(T x, T y) \leq \alpha d(x, y) .
$$

Mizoguchi and Takahashi [5] had defined a MT-function as follows: 
Definition 5. [16] A function $\varphi:[0,+\infty) \rightarrow[0,1)$ is said to be a MT-function if it satisfies Mizoguchi and Takahashi's condition (i.e., $\lim \sup _{r \rightarrow t^{+}} \varphi(r)<1$ for all $t \in[0,+\infty)$ ). Clearly, if $\varphi:[0,+\infty) \rightarrow[0,1)$ is a nondecreasing function or a nonincreasing function, then it is a MT-function.

Now we state some results from the basic theory of multivalued mappings.

Lemma 1. [17] Let $(X, d)$ be a metric space and $A, B \in C B(X)$, with $H(A, B)<\epsilon$, then for each $a \in A$, there exists an element $b \in B$ such that:

$$
d(a, b)<\epsilon .
$$

Lemma 2. [18] Let $(X, d)$ be a metric space and $A, B \in C B(X)$, then for each a $\in A$ :

$$
d(a, B) \leq H(A, B) .
$$

Lemma 3. [19] Let $\left\{A_{n}\right\}$ be a sequence in $C B(X)$ and there exists $A \in C B(X)$ such that $\lim _{n \rightarrow \infty} H\left(A_{n}, A\right) \rightarrow 0$. If $x_{n} \in A_{n}(n=1,2,3, \ldots)$ and there exists $x \in X$ such that $\lim _{n \rightarrow \infty} d\left(x_{n}, x\right) \rightarrow 0$ then $x \in A$.

\section{Main Results}

Definition 6. [20] A multivalued mapping $F: X \rightarrow C B(X)$ is said to be Mizoguchi-Takahashi G-contraction if for all $x, y$ in $X, x \neq y$ with $(x, y) \in E(G)$ :

(i) $H(F(x), F(y)) \leq \varphi(d(x, y)) d(x, y)$;

(ii) If $u \in F(x)$ and $v \in F(y)$ are such that $d(u, v) \leq d(x, y)$, then $(u, v) \in E(G)$.

Motivated by the Definition 2.1 of [20], in a more general settings, we define the sequence of multivalued $G_{f}$-contraction as follows:

Definition 7. Let $f: X \rightarrow X$ be a edge preserving surjection. A sequence of multivalued mappings $\left\{T_{q}\right\}_{q=1}^{\infty}$ from $X$ into $C B(X)$ is said to be sequence of multivalued $G_{f}$-contraction if $(f u, f v) \in E(G)$, implies:

$$
H\left(T_{q}(u), T_{r}(v)\right) \leq \mu(d(f u, f v)) d(f u, f v), \text { for all } q, r \in \mathbb{N} .
$$

For $x \in T_{q}(u)$ and $y \in T_{r}(v)$ satisfying $d(f x, f y) \leq d(f u, f v)$ implies $(f x, f y) \in E(G)$, where $\mu$ : $[0, \infty) \rightarrow[0,1)$ is a MT-function.

The next theorem provides the way to find the coincidence of a self map and a sequence of multivalued maps.

Theorem 1. Let $(X, d)$ a complete metric space, $\left\{T_{q}\right\}_{q=1}^{\infty}$ a sequence of multivalued $G_{f}$-contraction from $X$ into $C B(X)$ and $f: X \rightarrow X$ a surjection. If there exist $m \in \mathbb{N}$ and $v_{0} \in X$, such that:

(i) $T_{1}\left(v_{0}\right) \cap\left[f v_{0}\right]_{G}^{m} \neq \phi$;

(ii) For any sequence $\left\{v_{n}\right\}$ in $X$, if $v_{n} \rightarrow v$ and $v_{n} \in T_{n}\left(v_{n-1}\right) \cap\left[v_{n-1}\right]_{G}^{m}$ for all $n \in \mathbb{N}$, then there exists $a$ subsequence $\left\{v_{n_{k}}\right\}$ such that $\left(v_{n_{k}}, v\right) \in E(G)$ for all $k \in \mathbb{N}$.

Then $f$ and sequence of mappings $\left\{T_{q}\right\}_{q=1}^{\infty}$ have a coincidence point, i.e., there exists $v^{*} \in X$ such that $f v^{*} \in \bigcap_{q \in \mathbb{N}} T_{q}\left(v^{*}\right)$.

Proof. Choose any $v_{1} \in X$ such that $f v_{1} \in T_{1}\left(v_{0}\right) \cap\left[f v_{0}\right]_{G}^{m}$ then there exists a path from $f v_{0}$ to $f v_{1}$, i.e., $f v_{0}=f u_{0}^{(1)}, \ldots f u_{m}^{(1)}=f v_{1} \in T_{1}\left(v_{0}\right)$, and $\left(f u_{i}^{(1)}, f u_{i+1}^{(1)}\right) \in E(G)$ for all $i=0,1,2, \ldots, m-1$. 
Without any loss of generality, assume that $f u_{k}^{(1)} \neq f u_{j}^{(1)}$ for each $k, j \in\{0,1,2, \ldots, m\}$ with $k \neq j$. Since $\left(f u_{0}^{(1)}, f u_{1}^{(1)}\right) \in E(G)$, so:

$$
\begin{aligned}
H\left(T_{1}\left(u_{0}^{(1)}\right), T_{2}\left(u_{1}^{(1)}\right)\right) & \leq \mu\left(d\left(f u_{0}^{(1)}, f u_{1}^{(1)}\right)\right) d\left(f u_{0}^{(1)}, f u_{1}^{(1)}\right) \\
& <\sqrt{\mu\left(d\left(f u_{0}^{(1)}, f u_{1}^{(1)}\right)\right)} d\left(f u_{0}^{(1)}, f u_{1}^{(1)}\right) \\
& <d\left(f u_{0}^{(1)}, f u_{1}^{(1)}\right)
\end{aligned}
$$

Rename $f v_{1}$ as $f u_{0}^{(2)}$. As $f u_{0}^{(2)} \in T_{1}\left(u_{0}^{(1)}\right)$, and using Lemma 1 one can find some $f u_{1}^{(2)} \in T_{2}\left(u_{1}^{(1)}\right)$ such that:

$$
d\left(f u_{0}^{(2)}, f u_{1}^{(2)}\right)<d\left(f u_{0}^{(1)}, f u_{1}^{(1)}\right)
$$

Since $\left(f u_{1}^{(1)}, f u_{2}^{(1)}\right) \in E(G)$, so:

$$
\begin{aligned}
H\left(T_{2}\left(u_{1}^{(1)}\right), T_{2}\left(u_{2}^{(1)}\right)\right) & \leq \mu\left(d\left(f u_{1}^{(1)}, f u_{2}^{(1)}\right)\right) d\left(f u_{1}^{(1)}, f u_{2}^{(1)}\right) \\
& <d\left(f u_{1}^{(1)}, f u_{2}^{(1)}\right) .
\end{aligned}
$$

Similarly since $f u_{1}^{(2)} \in T_{2}\left(u_{1}^{(1)}\right)$, again using Lemma 1 one can find some $f u_{2}^{(2)} \in T_{2}\left(u_{2}^{(1)}\right)$ such that:

$$
d\left(f u_{1}^{(2)}, f u_{2}^{(2)}\right)<d\left(f u_{1}^{(1)}, f u_{2}^{(1)}\right)
$$

Thus we obtain $\left\{f u_{0}^{(2)}, f u_{1}^{(2)}, f u_{2}^{(2)}, \ldots, f u_{m}^{(2)}\right\}$ of $m+1$ vertices of $X$ such that $f u_{0}^{(2)} \in T_{1}\left(u_{0}^{(1)}\right)$ and $f u_{s}^{(2)} \in T_{2}\left(u_{s}^{(1)}\right)$ for $s=1,2, \ldots, m$, with:

$$
d\left(f u_{s}^{(2)}, f u_{s+1}^{(2)}\right)<d\left(f u_{s}^{(1)}, f u_{s+1}^{(1)}\right)
$$

for $s=0,1,2, \ldots, m-1$. As $\left(f u_{s}^{(1)}, f u_{s+1}^{(1)}\right) \in E(G)$ for all $s=0,1,2, \ldots, m-1$, thus $\left(f u_{s}^{(2)}, f u_{s+1}^{(2)}\right) \in$ $E(G)$ for all $s=0,1,2, \ldots, m-1$.

Let $f u_{m}^{(2)}=f v_{2}$. Thus the set of points $f v_{1}=f u_{0}^{(2)}, f u_{1}^{(2)}, f u_{2}^{(2)}, \ldots, f u_{m}^{(2)}=f v_{2} \in T_{2}\left(v_{1}\right)$ is a path from $f v_{1}$ to $f v_{2}$. Rename $f v_{2}$ as $f u_{0}^{(3)}$. Then by the same procedure we obtain a path:

$$
f v_{2}=f u_{0}^{(3)}, f u_{1}^{(3)}, f u_{2}^{(3)}, \cdots, f u_{m}^{(3)}=f v_{3} \in T_{3}\left(v_{2}\right)
$$

from $f v_{2}$ to $f v_{3}$. Inductively, obtained:

$$
f v_{h}=f u_{0}^{(h+1)}, f u_{1}^{(h+1)}, f u_{2}^{(h+1)}, \ldots, f u_{m}^{(h+1)}=f v_{h+1} \in T_{h+1}\left(v_{h}\right)
$$

with:

$$
d\left(f u_{t}^{(h+1)}, f u_{t+1}^{(h+1)}\right)<d\left(f u_{t}^{(h)}, f u_{t+1}^{(h)}\right)
$$

hence $\left(f u_{t}^{(h+1)}, f u_{t+1}^{(h+1)}\right) \in E(G)$ for $t=0,1,2, \ldots, m-1$.

Consequently, construct a sequence $\left\{f v_{h}\right\}_{h=1}^{\infty}$ of points of $X$ with:

$$
\begin{aligned}
f v_{1}= & f u_{m}^{(1)}=f u_{0}^{(2)} \in T_{1}\left(v_{0}\right), \\
f v_{2}= & f u_{m}^{(2)}=f u_{0}^{(3)} \in T_{2}\left(v_{1}\right), \\
f v_{3}= & f u_{m}^{(3)}=f u_{0}^{(4)} \in T_{3}\left(v_{2}\right), \\
& \vdots \\
f v_{h+1}= & f u_{m}^{(h+1)}=f u_{0}^{(h+2)} \in T_{h+1}\left(v_{h}\right),
\end{aligned}
$$


for all $h \in \mathbb{N}$.

For each $t \in\{0,1,2, \ldots, m-1\}$, and from (2), clearly $\left\{d\left(f u_{t}^{(h)}, f u_{t+1}^{(h)}\right)\right\}_{h=1}^{\infty}$ is a decreasing sequence of non-negative real numbers and so there exists $a_{t} \geq 0$ such that:

$$
\lim _{h \rightarrow \infty} d\left(f u_{t}^{(h)}, f u_{t+1}^{(h)}\right)=a_{t} .
$$

By assumption, $\lim \sup _{t \rightarrow a_{t}^{+}} \mu(t)<1$, so there exists $k_{t} \in \mathbb{N}$ such that $\mu\left(d\left(f u_{t}^{(h)}, f u_{t+1}^{(h)}\right)\right)<\omega\left(a_{t}\right)$ for all $h \geq k_{t}$ where $\limsup _{t \rightarrow a_{t}^{+}} \mu(t)<\omega\left(a_{t}\right)<1$.

Now put:

$$
\Theta_{t}=\max \left\{\max _{r=1, \ldots, k_{t}} \sqrt{\mu\left(d\left(f u_{t}^{(r)}, f u_{t+1}^{(r)}\right)\right)}, \sqrt{\omega\left(a_{t}\right)}\right\} .
$$

Then, for every $h>k_{t}$, consider:

$$
\begin{aligned}
d\left(f u_{t}^{(h+1)}, f u_{t+1}^{(h+1)}\right) & <\sqrt{\mu\left(d\left(f u_{t}^{(h)}, f u_{t+1}^{(h)}\right)\right)} d\left(f u_{t}^{(h)}, f u_{t+1}^{(h)}\right) \\
& <\sqrt{\omega\left(a_{t}\right)} d\left(f u_{t}^{(h)}, f u_{t+1}^{(h)}\right) \\
& \leq \Theta_{t} d\left(f u_{t}^{(h)}, f u_{t+1}^{(h)}\right) \\
& \leq\left(\Theta_{t}\right)^{2} d\left(f u_{t}^{(h-1)}, f u_{t+1}^{(h-1)}\right) \\
& \leq \cdots \\
& \leq\left(\Theta_{t}\right)^{h} d\left(f u_{t}^{(1)}, f u_{t+1}^{(1)}\right) .
\end{aligned}
$$

Putting $q=\max \left\{k_{t}: t=0,1,2, \ldots, m-1\right\}$, gives:

$$
\begin{aligned}
d\left(f v_{h}, f v_{h+1}\right) & =d\left(f u_{0}^{(h+1)}, f u_{m}^{(h+1)}\right) \\
& \leq \sum_{t=0}^{m-1} d\left(f u_{t}^{(h+1)}, f u_{t+1}^{(h+1)}\right) \\
& <\sum_{t=0}^{m-1}\left(\Theta_{t}\right)^{h} d\left(f u_{t}^{(1)}, f u_{t+1}^{(1)}\right), \text { for all } h>q .
\end{aligned}
$$

Now for $p>h>q$, consider:

$$
\begin{aligned}
d\left(f v_{h}, f v_{p}\right) & \leq d\left(f v_{h}, f v_{h+1}\right)+d\left(f v_{h+1}, f v_{h+2}\right)+\cdots+d\left(f v_{p-1}, f v_{p}\right) \\
& <\sum_{t=0}^{m-1}\left(\Theta_{t}\right)^{h} d\left(f u_{t}^{(1)}, f u_{t+1}^{(1)}\right)+\cdots+\sum_{t=0}^{m-1}\left(\Theta_{t}\right)^{p-1} d\left(f u_{t}^{(1)}, f u_{t+1}^{(1)}\right) .
\end{aligned}
$$

Since $\Theta_{t}<1$ for all $t \in\{0,1,2, \ldots, m-1\}$, it follows that $\left\{f v_{h}=f u_{m}^{(h)}\right\}$ is a Cauchy sequence. Using completeness of $X$, find $v^{*} \in X$ such that $f v_{h} \rightarrow f v^{*}$. Now using the fact that $f v_{n} \in T\left(v_{n-1}\right) \cap$ $\left[f v_{n-1}\right]_{G}^{m}$ for all $n \in \mathbb{N}$, find a subsequence $\left\{f v_{n_{k}}\right\}$ of $\left\{f v_{h}\right\}$ such that $\left(f v_{n_{k}}, f v^{*}\right) \in E(G)$ for all $k \in \mathbb{N}$. Now for any $q \in \mathbb{N}$ :

$$
\begin{aligned}
d\left(f v^{*}, T_{q}\left(v^{*}\right)\right) & \leq d\left(f v^{*}, f v_{h+1}\right)+d\left(f v_{h+1}, T_{q}\left(v^{*}\right)\right) \\
& \leq d\left(f v^{*}, f v_{h+1}\right)+H\left(T_{h+1}\left(v_{h}\right), T_{q}\left(v^{*}\right)\right) \\
& \leq d\left(f v^{*}, f v_{h+1}\right)+\mu\left(d\left(f v_{h}, f v^{*}\right)\right) d\left(f v_{h}, f v^{*}\right) .
\end{aligned}
$$

Letting $h \rightarrow \infty$ in the above inequality, gives $d\left(f v^{*}, T_{q}\left(v^{*}\right)\right) \rightarrow 0$, which implies $f v^{*} \in T_{q}\left(v^{*}\right)$ for all $q \in \mathbb{N}$. Hence, $f v^{*} \in \bigcap_{q \in \mathbb{N}} T_{q}\left(v^{*}\right)$ as required. 
Example 1. Let $X=\{0\} \cup\left\{\frac{1}{q^{n}}: n \in \mathbb{N} \cup\{0\}\right\}$ for $q \in \mathbb{N}$. Consider the graph $G$ such that $V(G)=X$ and for all $x$ and $y$ in $X$ :

$$
E(G)=\{(x, y): x \neq y\} .
$$

For $q \in \mathbb{N}$, let $T_{q}: X \rightarrow C B(X)$ be defined by:

$$
T_{q}(x)=\left\{\begin{array}{cc}
\left\{0, \frac{1}{q}+1,1\right\} & \text { if } x=0, \\
\left\{\frac{1}{q^{n+1}}+1,1\right\} & \text { if } x=\frac{1}{q^{n}}, n \in \mathbb{N}, \\
\left\{\frac{1}{q}+1\right\} & \text { if } x=1 .
\end{array}\right.
$$

If we assume $f: X \rightarrow X$ as an identity map then sequence of multivalued mappings $\left\{T_{q}\right\}_{q=1}^{\infty}$ from $X$ into $C B(X)$ is a sequence of multivalued $G_{f}$-contraction.

It satisfies the conditions of Theorem 1 and $1 \in X$ is the fixed point of sequence of multivalued maps $T_{q}$ for $q \in \mathbb{N}$.

The next theorem provides a way to find the coincidence point of a hybrid pair.

Theorem 2. Let $(X, d)$ be a complete metric space, $T: X \rightarrow C B(X)$ and $f: X \rightarrow X$ a surjection. If $u, v \in X($ with $u \neq v)$ such that $(f u, f v) \in E(G)$, implies:

$$
H(T(u), T(v)) \leq \mu(d(f u, f v)) d(f u, f v),
$$

where $\mu:[0, \infty) \rightarrow[0,1)$ is a MT-function, if there exist $m \in \mathbb{N}$ and $v_{0} \in X$, such that:

(i) $T\left(v_{0}\right) \cap\left[f v_{0}\right]_{G}^{m} \neq \phi$;

(ii) For any sequence $\left\{v_{n}\right\}$ in $X$, if $v_{n} \rightarrow v$ and $v_{n} \in T\left(v_{n-1}\right) \cap\left[v_{n-1}\right]_{G}^{m}$ for all $n \in \mathbb{N}$ and $j=1,2, \ldots$, then there exists a subsequence $\left\{v_{n_{k}}\right\}$ such that $\left(v_{n_{k}}, v\right) \in E(G)$ for all $k \in \mathbb{N}$.

Then $f$ and $T$ have a coincidence point, i.e., there exists $v^{*} \in X$ such that $f v^{*} \in T\left(v^{*}\right)$.

Proof. Take $T_{q}:=T$ for all $q \in \mathbb{N}$ in Theorem 1 and proof is following the same procedure.

Corollary 1. Let $(X, d)$ be a complete metric space, $\left\{T_{q}\right\}_{q=1}^{\infty}$ a sequence of the self mappings on $X$ and $f: X \rightarrow X$ a surjection. If $u, v \in X($ with $u \neq v)$ such that $(f u, f v) \in E(G)$, implies:

$$
d\left(T_{q}(u), T_{r}(v)\right) \leq \mu(d(f u, f v)) d(f u, f v),
$$

for all $q, r \in \mathbb{N}$, where $\mu:[0, \infty) \rightarrow[0,1)$ is a MT function, if there exist $m \in \mathbb{N}$ and $v_{0} \in X$, such that:

(i) $T_{1}\left(v_{0}\right) \cap\left[f v_{0}\right]_{G}^{m} \neq \phi$;

(ii) For any sequence $\left\{v_{n}\right\}$ in $X$, if $v_{n} \rightarrow v$ and $v_{n}=T_{n}\left(v_{n-1}\right) \cap\left[v_{n-1}\right]_{G}^{m}$ for all $n \in \mathbb{N}$,

then there exists a subsequence $\left\{v_{n_{k}}\right\}$ such that $\left(v_{n_{k}}, v\right) \in E(G)$ for all $k \in \mathbb{N}$.

Then $f$ and sequence of mappings $\left\{T_{q}\right\}_{q=1}^{\infty}$ have a coincidence point, i.e., there exists $v^{*} \in X$ such that $f v^{*}=\bigcap_{q \in \mathbb{N}} T_{q}\left(v^{*}\right)$.

Corollary 2. Let $(X, d)$ be a complete metric space, $T: X \rightarrow C B(X)$ and if $u, v \in X$ (with $u \neq v)$ such that $(u, v) \in E(G)$, implies:

$$
H(T(u), T(v)) \leq \mu(d(u, v)) d(u, v),
$$

where $\mu:[0, \infty) \rightarrow[0,1)$ is a MT-function, if there exist $m \in \mathbb{N}$ and $v_{0} \in X$, such that:

(i) $T\left(v_{0}\right) \cap\left[v_{0}\right]_{G}^{m} \neq \phi$; 
(ii) For any sequence $\left\{v_{n}\right\}$ in $X$, if $v_{n} \rightarrow v$ and $v_{n} \in T\left(v_{n-1}\right) \cap\left[v_{n-1}\right]_{G}^{m}$ for all $n \in \mathbb{N}$ and $j=1,2, \ldots$, then there exists a subsequence $\left\{v_{n_{k}}\right\}$ such that $\left(v_{n_{k}}, v\right) \in E(G)$ for all $k \in \mathbb{N}$.

Then $T$ has a fixed point, i.e., $v^{*}=T\left(v^{*}\right)$.

The following are the consequence of the Theorem 1 and Theorem 2 for the case of self mappings.

Corollary 3. Let $(X, d)$ be a complete metric space, $T: X \rightarrow X$ and $f: X \rightarrow X$ a surjection. If $u, v \in X($ with $u \neq v)$ such that $(f u, f v) \in E(G)$, implies:

$$
d(T(u), T(v)) \leq \mu(d(f u, f v)) d(f u, f v)
$$

where $\mu:[0, \infty) \rightarrow[0,1)$ is a MT function, if there exist $m \in \mathbb{N}$ and $v_{0} \in X$, such that:

(i) $T\left(v_{0}\right) \cap\left[f v_{0}\right]_{G}^{m} \neq \phi$;

(ii) For any sequence $\left\{v_{n}\right\}$ in $X$, if $v_{n} \rightarrow v$ and $v_{n}=T\left(v_{n-1}\right) \cap\left[v_{n-1}\right]_{G}^{m}$ for all $n \in \mathbb{N}$ and $j=1,2, \ldots$, then there exists a subsequence $\left\{v_{n_{k}}\right\}$ such that $\left(v_{n_{k}}, v\right) \in E(G)$ for all $k \in \mathbb{N}$.

Then $f$ and $T$ have a coincidence point, i.e., there exists $v^{*} \in X$ such that $f v^{*}=T\left(v^{*}\right)$.

Corollary 4. Let $(X, d)$ be a complete metric space, $T: X \rightarrow X$ and if $u, v \in X($ with $u \neq v)$ such that $(u, v) \in E(G)$, implies:

$$
d(T(u), T(v)) \leq \mu(d(u, v)) d(u, v),
$$

where $\mu:[0, \infty) \rightarrow[0,1)$ is a MT-function, if there exist $m \in \mathbb{N}$ and $v_{0} \in X$, such that:

(i) $T\left(v_{0}\right) \cap\left[v_{0}\right]_{G}^{m} \neq \phi$;

(ii) For any sequence $\left\{v_{n}\right\}$ in $X$, if $v_{n} \rightarrow v$ and $v_{n}=T\left(v_{n-1}\right) \cap\left[v_{n-1}\right]_{G}^{m}$ for all $n \in \mathbb{N}$ and $j=1,2, \ldots$, then there exists a subsequence $\left\{v_{n_{k}}\right\}$ such that $\left(v_{n_{k}}, v\right) \in E(G)$ for all $k \in \mathbb{N}$.

Then $T$ has a fixed point, i.e., $v^{*}=T\left(v^{*}\right)$.

The next remark highlights the applications of all the above results in settings of complete metric spaces, complete metric spaces endowed with partial order and $\varepsilon$-chainable complete metric spaces.

Remark 1. Consider the following cases:

R1. Let $(X, d)$ be a complete metric space, consider the graph $G_{0}$ with:

$$
E\left(G_{0}\right)=X \times X
$$

R2. Let $(X, d)$ be a complete metric space with partial order $\preceq$ on $X$, consider the graphs $G_{1}$ and $G_{2}$ with:

$$
E\left(G_{1}\right)=\{(x, y) \in X \times X: x \preceq y\}
$$

and:

$$
E\left(G_{2}\right)=\{(x, y) \in X \times X: x \preceq y \text { or } y \preceq x\}
$$

R3. Let $\varepsilon>0$ and $(X, d)$ be a complete $\varepsilon$-chainable metric space, consider the graph:

$$
G_{3}:=\{(x, y) \in X \times X: 0<d(x, y)<\varepsilon, \text { for } \varepsilon>0\} \text {. }
$$

We remark that all above results are valid under the above construction of remarks $(R 1),(R 2)$ and $(R 3)$.

Further, in an application of Theorem 1 we generalize the Theorem 6 of [20]. It establishes the convergence of successive approximations of operators on a Banach space, which consequently yields 
the Kelisky-Rivlin theorem on iterates of Bernstein operators on the space $C(I)$, where $I$ is the closed unit interval.

Theorem 3. Let $X$ be a Banach space and $X_{0}$ be a closed subspace of $X$. Let $T, f: X \rightarrow X$ be maps such that $f$ is surjection and:

$$
\|T x-T y\| \leq \varphi(\|f x-f y\|)\|f x-f y\| \text { whenever } f x-f y \in X_{0}, x \neq y .
$$

If $(I-f)(X) \subseteq X_{0}$ and $(f-T)(X) \subseteq X_{0}$, then for all $x \in X,\left\{T^{n} x\right\}$ converges to Coin $\{T, f\}$, where $\operatorname{Coin}\{T, f\}=\{x \in X: T x=f x\}$.

Proof. Consider the graph $G=(V(G), E(G))$ where $V(G)=X$ and $E(G)=$ $\left\{(x, y) \in X \times X: x-y \in X_{0}\right\}$. Clearly, $\triangle \subseteq E(G), \tilde{G}=G$ and $G$ has no parallel edges. Consider $(x, y) \in E(G)$, then $f x-f y=(y-f y)-(x-f x)+(x-y) \in X_{0}$, since $(I-f)(X) \subseteq X_{0}$. Hence and by given contractive condition (9), we see that $\forall(x, y) \in E(G)$ with $x \neq y$, (6) holds. Also $T x-T y=(f y-T y)-(f x-T x)+(f x-f y) \in X_{0}$, since $(f-T)(X) \subseteq X_{0}$.

The use of $(f-T)(X) \subseteq X_{0}$, implies that $(f x, T x) \in E(G)$ for $x$ in $X$. Therefore condition $(i)$ of Corollary 4 holds with $x=v_{0}=x_{0}$ and $N=1$. Thus we are able to generate a sequence such that $T x_{n-1}=f x_{n}$ for all $n \in \mathbb{N}$. Assume that $T x_{n} \rightarrow v^{*} \in X$ but since $f$ is surjection so there exists some $v$ in $X$ such that $v^{*}=f v$. Here also $T x_{n} \in\left[T x_{n-1}\right]_{G}^{1}$ for all $n \in \mathbb{N}$, which implies that $\left(T x_{n}, T x_{n-1}\right) \in E(G)$ for all $n \in \mathbb{N}$. Now using the outline of the proof of Theorem 4.1 of [12], $\left(T x_{n}, f v\right) \in E(G)$ for all $n \in \mathbb{N}$. Now assume:

$$
\begin{aligned}
\|f v-T v\| & \leq\left\|f v-f x_{n+1}\right\|+\left\|f x_{n+1}-T v\right\| \\
& =\left\|f v-f x_{n+1}\right\|+\left\|T x_{n}-T v\right\| .
\end{aligned}
$$

Since $\left(T x_{n}, f v\right) \in E(G)$ for all $n \in \mathbb{N}$, thus from (9) and (10) we have:

$$
\|f v-T v\| \leq\left\|f v-f x_{n+1}\right\|+\varphi\left(\left\|f x_{n}-f v\right\|\right)\left\|f x_{n}-f v\right\| .
$$

As $n \rightarrow \infty$, we get $f v=T v$. Thus $v$ is the coincidence point of $f$ and $T$, by using Corollary 4 . For the uniqueness of the coincidence point we let two coincidence points $u, v$ of $f$ and $T$, then:

$$
\begin{aligned}
\|T u-T v\| & \leq \varphi(\|f u-f v\|)\|f u-f v\| \\
(1-\varphi(\|f u-f v\|))\|T u-T v\| & \leq 0 .
\end{aligned}
$$

This implies that $T u=T v$.

In the next result, we discussed the generalization of fractional differential equation described in [21]. For the closed interval $I=[0,1]$, assume function $g \in C(I, \mathbb{R})$ and $f: I \times \mathbb{R} \rightarrow \mathbb{R}$ is a continuous function. The fractional differential equation is given as follows:

$$
D^{\alpha} x(t)+f(t, g(x(t)))=0 \quad(0 \leq t \leq 1, \alpha>1)
$$

with boundary conditions $x(0)=x(1)=0$. It is to be noted that associated Green's function with the problem (11) is:

$$
G(t, s)=\left\{\begin{array}{ll}
(t(1-s))^{\alpha-1}-(t-s)^{\alpha-1} & 0 \leq s \leq t \leq 1, \\
\frac{(t(1-s))^{\alpha-1}}{\Gamma(\alpha)} & 0 \leq t \leq s \leq 1 .
\end{array},\right.
$$

where $\Gamma($.$) represents the Gamma function.$

Theorem 4. Consider the surjective function $g \in C(I, \mathbb{R})$ and $f: I \times \mathbb{R} \rightarrow \mathbb{R}$ satisfies:

(i) $|(f(s, g(x(s)))-f(s, g(y(s))))| \leq|g(x(s))-g(y(s))|$ for all $s \in I$; 
(ii) $\sup _{t \in I} \int_{0}^{1} G(t, s) d s \leq k<1$.

Then, problem (11) has a unique solution.

Proof. Assume space $X=C(I, \mathbb{R})$, and we have $d(x, y)=\max _{t \in[0,1]}|x(t)-y(t)|$ for $x$ and $y$ in $X$. It is well known that $x \in X$ is a solution of (11) if and only if it is a solution of the integral equation:

$$
x(t)=\int_{0}^{1} G(t, s) f(s,(g x)(s)) d s \text { for all } t \in I .
$$

Define the operator $F: X \rightarrow X$ by:

$$
F x(t)=\int_{0}^{1} G(t, s) f(s,(g x)(s)) d s \text { for all } t \in I,
$$

and $S: X \rightarrow X$ by:

$$
S x=g x, \text { with }(S x)(t)=(g x)(t) \text { for } t \in I .
$$

Thus, for finding a solution of (11), it is sufficient to show that $F$ has a coincidence point with $g$. Now let $x, y \in C(I)$ for all $s \in I$. Here we have:

$$
\begin{aligned}
|F x(t)-F y(t)| & =\left|\int_{0}^{1} G(t, s)(f(s,(g x)(s))-f(s,(g y)(s))) d s\right| \\
& \leq \int_{0}^{1} G(t, s)|(f(s,(g x)(s))-f(s,(g y)(s)))| d s \\
& \leq \int_{0}^{1} G(t, s)|(g x)(s)-(g y)(s)| d s \\
& \leq \int_{0}^{1} G(t, s)|(S x)(s)-(S y)(s)| d s \\
& \leq \int_{0}^{1} G(t, s) d(S x, S y) d s \\
& \leq d(S x, S y) \sup _{t \in I}^{1} G(t, s) d s \\
& \leq k d(S x, S y) .
\end{aligned}
$$

This implies that for each $x, y \in X$, we have:

$$
d(F x, F y) \leq k d(S x, S y) .
$$

Now the use of Corollary 3 with graph $G=G_{0}$, we have $x^{*} \in X$ such that $F x^{*}=S x^{*}$ with $\left(S x^{*}\right)(t)=\left(g x^{*}\right)(t)$ for $t \in I$. Thus $x^{*}$ is the required coincidence point of $F$ and $g$.

Author Contributions: All authors contributed equally to the writing of this paper. All authors read and approve the final manuscript. 
Conflicts of Interest: The authors declare no conflict of interest.

\section{References}

1. Nadler, B. Multivalued contraction mappings. Pacific J. Math. 1969, 30, 475-488.

2. Hu, T. Fixed point theorems for multivalued mappings. Can. Math. Bull. 1980, 23, 193-197.

3. Covitz, H.; Nadler, SB, Jr. Multi-valued contraction mappings in generalized metric spaces. Israel J. Math. 1970, 8, 5-11.

4. Reich, S. Fixed points of contractive functions. Boll. Unione Mat. Ital. 1972, 5, 26-42.

5. Mizoguchi, N.; Takahashi, W. Fixed point theorems for multivalued mappings on complete metric spaces. J. Math. Anal. Appl. 1989, 141, 177-188.

6. Azam, A.; Arshad, M. Fixed points of a sequence of locally contractive multivalued maps. Comp. Math. Appl. 2009, 57, 96-100.

7. Feng, Y.; Liu, S. Fixed point theorems for multi-valued contractive mappings and multi-valued Caristi type mappings. J. Math. Anal. Appl. 2006, 317, 103-112.

8. Ciric, L.B. Multi-valued nonlinear contraction mappings. Nonlinear Anal. 2009, 71, 2716-2723.

9. Klim, D.; Wardowski, D. Fixed point theorems for set-valued contractions in complete metric spaces. J. Math. Anal. Appl. 2007, 334, 132-139.

10. Klim, D.; Wardowski, D. Fixed points of dynamic processes of set-valued F-contractions and application to functional equations. Fixed Point Theory Appl. 2015, 1, 22.

11. Nicolae, A. Fixed point theorems for multi-valued mappings of Feng-Liu type. Fixed Point Theory 2011, 12, 145-154.

12. Jachymski, J. The contraction principle for mappings on a metric space with a graph. Proc. Amer. Math. Soc. 2008, 136, 1359-1373.

13. Nieto, J.; Rodríguez-López, R. Contractive mapping theorems in partially ordered sets and applications to ordinary differential equations. Order 2005, 3, 223-239.

14. Ran, A.C.M.; Reurings, M.C.B. A fixed point theorem in partially ordered sets and some applications to matrix equations. Proc. Am. Math. Soc. 2004, 1435-1443.

15. Johnsonbaugh, R. Discrete Mathematics; Prentice-Hall, Inc.: Upper Saddle River, NJ, USA, 1997.

16. Du, W.S. On coincidence point and fixed point theorems for nonlinear multivalued maps. Topol. Appl. 2012, $159,49-56$.

17. Hu, T.; Rosen, H. Locally contractive and expansive mappings. Boll. Unione Mat. Ital. 1990, 7, $227-233$.

18. Bailey, B.F. Some theorems on contractive mappings. J. Lond. Math. Soc. 1966, 41, 101-106.

19. Assad, N.A.; Kirk, W.A. Fixed point theorems for set valued mappings of contractive type. Pacific J. Math. 1972, 43, 533-562.

20. Asrifa, S.; Vetrivel, V. Fixed points of Mizoguchi-Takahashi contraction on metric space with a graph and applications. J. Math. Anal. Appl. 2014, 417, 336-344.

21. Baleanu, D.; Shahram, R.; Hakimeh, M. Some existence results on nonlinear fractional differential equations. Philos. Trans. A Math. Phys. Eng. Sci. 2013, 371, 20120144.

(C) 2017 by the authors. Licensee MDPI, Basel, Switzerland. This article is an open access article distributed under the terms and conditions of the Creative Commons Attribution (CC BY) license (http://creativecommons.org/licenses/by/4.0/). 